\title{
All-in-One: VQ-VAE for End-to-End Joint Source-Channel Coding
}

This paper was downloaded from TechRxiv (https://www.techrxiv.org).

LICENSE

CC BY-NC-SA 4.0

SUBMISSION DATE / POSTED DATE

02-03-2022 / 07-03-2022

\section{CITATION}

Nemati, Mahyar; Choi, Jinho (2022): All-in-One: VQ-VAE for End-to-End Joint Source-Channel Coding. TechRxiv. Preprint. https://doi.org/10.36227/techrxiv.19294622.v1

$\mathrm{DOI}$

10.36227/techrxiv.19294622.v1 


\title{
All-in-One: VQ-VAE for End-to-End Joint Source-Channel Coding
}

\author{
Mahyar Nemati and Jinho Choi
}

\begin{abstract}
Vector Quantized Variational Autoencoder (VQ-VAE) has been regarded as a promising representation of diverse and complex data distributions in deep learning ecosystem. However, its use in a systematic way leveraging existing wireless communications has not been well addressed. In this paper, we explore the VQ-VAE characteristics in a point-to-point wireless communication and modify its training process to design a joint source-channel coding that is robust against noisy wireless channels. With all due respect to the source-channel separation theorem, various factors prevent error-free transmissions of conventional coding schemes in reality. Likewise, the proposed model is not error-free, but it compromises the reliability and complexity of the system. Thus, the proposed model makes the physical/link layer lighter while preserving reliability. It is considered an alternative for further data compression compared to the conventional separated source-channel coding schemes. Our system has been evaluated with extensive simulations, providing insightful observations and findings.
\end{abstract}

\section{Index Terms}

Lossy Compression; VQ-VAE; Noisy Channels

\section{INTRODUCTION}

\section{A. Background}

For point-to-point communication over a noisy channel, it is well-known that source coding and channel coding can be performed separately, which is called the source-channel separation theorem [1], [2]. According to this theorem, it is possible to design a pair of source encoder (ENC) and source decoder (DEC) by taking into account the statistical properties of a source as if the channel is error-free. Then, to mitigate the noise, interference, and distortion, a pair of channel ENC and channel DEC can be designed for a given channel.

\section{B. Data Compression using Neural Networks}

For source coding, when the size of data samples is large, data compression, which is also known as dimensionality reduction, is an appropriate process of reducing the redundancy. There are various approaches to source coding. Most approaches are based on a statistical model of source (e.g., the distribution), but often there is no statistical model and only data samples are available. Motivated by this, it is possible to use variational-auto-encoder (VAE) [3] to compress a high-dimensional signal into a low-dimensional signal. The VAEs use neural networks (NN) architectures composed of an ENC and a DEC. The

The authors are with the School of Information Technology, Deakin University, Geelong, VIC 3220, Australia (e-mail: [n.mahyar, jinho.choi]@ deakin.edu.au). This research was supported by the Australian Government through the Australian Research Council's Discovery Projects funding scheme (DP200100391).

This work has been submitted to the IEEE for possible publication. Copyright may be transferred without notice, after which this version may no longer be accessible. 
ENC is connected to the DEC via a latent space. Latent space is a compressed representation of data and contains a specific number of continuous latent vectors, each with a unique dimension. Since it is generally harder to learn such continuous vector distributions via gradient descent in deep learning, Vector Quantization (VQ) technique is taken into account in the VAE which is known as VQ-VAE [4]. The VQ is used as a quantization technique to generate some codewords for representing the continuous latent vectors of the VAE. It makes the optimization problem much simpler.

\section{Scope, Novelty, Contributions}

Suppose conventional VQ-VAE as an integrated end-to-end lossy compression design. We aim to split the ENC and DEC for a transmitter $(\mathrm{Tx})$ and a receiver $(\mathrm{Rx})$, respectively, in a point-to-point communication over noisy wireless channels. We model the noisy wireless link between the ENC and DEC by a discrete memoryless channel (DMC). Accordingly, we modify the training of VQ-VAE to adapt it to the proposed wireless communication scenario for the first time. To be more specific, each codeword is represented by an index of binary digits used in digital communication systems [5], [6]. Then, the ENC and DEC learn the channel impact on these binary digits that makes the system robust against the noisy channels. Indeed, it is concluded that the ENC and the DEC learn a joint source-channel coding in the deep learning ecosystem.

We note that it is different from the conventional separated source-channel coding schemes. With all due respect to the source-channel separation theorem [1], [2] and considering the imperfection of channel coding in reality (due to unknown varying fading in wireless communications), the proposed model compromises the reliability and complexity of the system. Indeed, the proposed model makes the physical/link layer lighter while preserving reliability. As a result, the proposed model can be considered an alternative for further data compression, with no source/channel statistics, compared to the conventional separated source-channel coding schemes. The main contributions and novelties of this study are summarized as follows:

- We explore the use of VQ-VAE in wireless communications for the first time. Then, we propose a joint source-channel coding using VQ-VAE which has a robust performance in noisy channels and results in a lighter physical/link layer in IoT networks.

- We split the ENC and DEC of a VQ-VAE and adapt its training within the noisy wireless channels. In other words, since the VQ-VAE is traditionally used for image compression only, its noisy channel robustness comes from harmonizing the latent space with the noisy wireless channels. Here, the noisy channel robustness in the deep learning ecosystem is associated with the traditional channel coding in physical layer domain. Thus, it is considered an integrated source-channel coding.

- We elaborate on the proposed data-driven model advantages as there is no need for source/channel statistics and the proposed system can be taken into action by enough datasets only. Furthermore, we provide extensive simulation results and justify our observations and findings. 


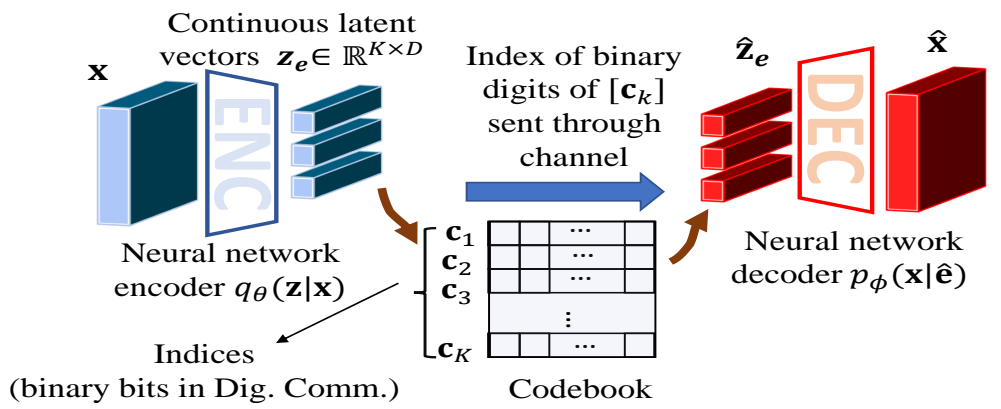

Fig. 1: Illustration of VQ-VAE in digital communication systems.

\section{VQ-VAE in Digital Communication Systems}

In this section, we elaborate on the VQ-VAE characteristics and its alignment within the wireless digital communication systems.

\section{A. Modeling of Source Coding}

Suppose there is a pair of a transmitter, Tx, and a receiver, Rx. As shown in Fig. 1, the Tx aims to transmit an extremely high-dimensional, i.e., $L$-dimensional, data, $\mathbf{x} \in \mathbb{R}^{L}$, where only its data samples $\mathbf{x}=\left[x_{1}, \cdots, x_{L}\right]$ are available and statistics of $\mathbf{x}$ are unknown. The Tx and Rx comprise an ENC and a DEC, respectively. Similar to the digital communication systems [5], [6], there is a shared codebook $\mathbf{C}=\left[\mathbf{c}_{1}, \cdots, \mathbf{c}_{K}\right] \in \mathbb{R}^{K \times D}$ which is a collection of $K, K \ll L$, codewords generated by the ENC, each of length $D$. The ENC first maps the high dimensional data input $\mathbf{x}$ to a continuous intermediate feature map output, $\mathbf{z}_{e}$, i.e., $\mathbf{x}$ as $\mathbf{x} \in \mathbb{R}^{L} \rightarrow \mathbf{z}_{e} \in \mathbb{R}^{K \times D}$, and then converts it to a discrete/quantized latent variable by choosing the nearest $\mathbf{c}_{k} \in \mathbf{C}$ codeword. Thus, the output of the ENC can be given by

$$
\mathbf{z}=\operatorname{ENC}(\mathbf{x})=k \quad \text { where } \quad \mathbf{c}_{k} \in \mathbf{C}=\left\{\mathbf{c}_{1}, \ldots, \mathbf{c}_{K}\right\}
$$

Basically, the ENC is the non-linear mapping from input space, $\mathbf{x}$, to the discrete latent embedded vectors, $\mathbf{c}_{k}$. Indeed the ENC is associated by a posterior categorical distribution $q_{\theta}(\mathbf{z} \mid \mathbf{x})$ as follows

$$
q_{\theta}(\mathbf{z}=k \mid \mathbf{x})= \begin{cases}1 & \text { if } k=\operatorname{argmin}_{j}\left\|\mathbf{z}_{e}-\mathbf{c}_{j}\right\|_{2} \\ 0 & \text { else }\end{cases}
$$

In other words, the ENC is trained to convert a high dimensional input into a sequence of continuous latent vectors, i.e., $\mathbf{z}_{e}$, and then to discretize them as

$$
\mathbf{z} \equiv \mathbf{c}_{k} \quad \text { where } \quad k=\underset{j}{\operatorname{argmin}}\left\|\mathbf{z}_{e}-\mathbf{c}_{j}\right\|_{2} .
$$

In the discretization process, we choose the nearest codeword, $\mathbf{c}_{k}$, based on the closest distance (e.g., L2-norm distance) from continuous representation $\mathbf{z}_{e}$. Eventually, each quantized vector is replaced with its index of binary digits of the nearest codebook vector, $k$, and is sent to the Rx for reconstruction. Thus, the length of messages in bits becomes $N=\left\lceil\log _{2} K\right\rceil$.

Different from the conventional digital communication systems [6], we do not access the statistical properties of $\mathbf{x}$ and it is the role of the ENC to learn the most important and semantically interesting features of the data in $\mathbf{z}_{e}$ [4]. 
At the Rx, to recover the original signal, the DEC is tasked with reconstructing data from the quantized vector indices. The DEC maps an index $k$ of a quantized vector from the shared codebook, $\mathbf{C}$, back to the original data, $\hat{\mathbf{x}}$, i.e., DEC : $\hat{\mathbf{z}}_{e} \in$ $\mathbb{R}^{K \times D} \rightarrow \hat{\mathbf{x}} \in \mathbb{R}^{L}$. That is, the output of the DEC is given by

$$
\hat{\mathbf{x}}=\operatorname{DEC}\left(\hat{\mathbf{z}}_{e}\right) \in \mathbb{R}^{L}
$$

\section{B. Imperfection of Channel Coding in Noisy Channels}

For the channel coding, while a channel code can be designed to ensure error-free transmissions over a noisy channel, there are various factors that prevent error-free transmissions. For example, as discussed in [7], the error probability cannot be zero for a finite-length code, although its code rate is less than the channel capacity. In addition, the channel parameters can vary (due to unknown fading in wireless communications), which may result in a decrease of the channel capacity and unsuccessful decoding.

Overall, in most well-established communication networks, source coding can take place in the application layer, while channel coding is performed in the physical layer. To ensure reliable transmissions, link-layer protocols such as automatic repeat request (ARQ) protocols [8] can be used above the physical layer. Thus, we conclude that transmission errors can occur in reality, which violates the conventional assumption of error-free transmissions for a pair of source ENC and DEC. As a result, using the lossy compression VQ-VAE as an alternative to conventional source-channel coding, we make the physical/link layer lighter for wireless sensor networks or Internet-of-Things (IoT) networks while preserving the reliability.

\section{Noisy Channel Interpretation in VQ-VAE}

When signals are compressed using VQ-VAE, the indices of the codewords are to be transmitted over a noisy channel. The length of messages in bits are $N$ and each message is to be encoded by a channel code of length $M$. As a result, the code rate becomes $R=\frac{N}{M}$. Denote by $\boldsymbol{\kappa}$ the index of codeword $\mathbf{c}_{\boldsymbol{\kappa}}$, which is referred to as the message. In addition, let $\mathbf{s}=$ CENC $(\boldsymbol{\kappa})$ be the output of a channel ENC. At the Rx, the received signal is given by

$$
\hat{\mathbf{s}}=h \sqrt{P} \mathbf{s}+\mathbf{n},
$$

where $\mathbf{n} \sim \mathcal{C N}\left(0, \sigma^{2} \mathbf{I}\right)$ is the background noise, $P$ is the signal power, and $h$ is the channel coefficient. We assume a blockfading channel [9], where the channel coefficient remains unchanged when a codeword $\mathbf{s}$ is transmitted. Letting $\alpha=|h|^{2}$ and assuming that $\mathbb{E}\left[\mathbf{s s}^{\mathrm{H}}\right]=\mathbf{I}$, the signal-to-noise ratio (SNR) becomes

$$
\gamma(\alpha)=\frac{\alpha P}{\sigma^{2}}
$$

When a capacity-achieving code is used, decoding becomes successful if

$$
R \leq \log _{2}(1+\gamma(\alpha))
$$




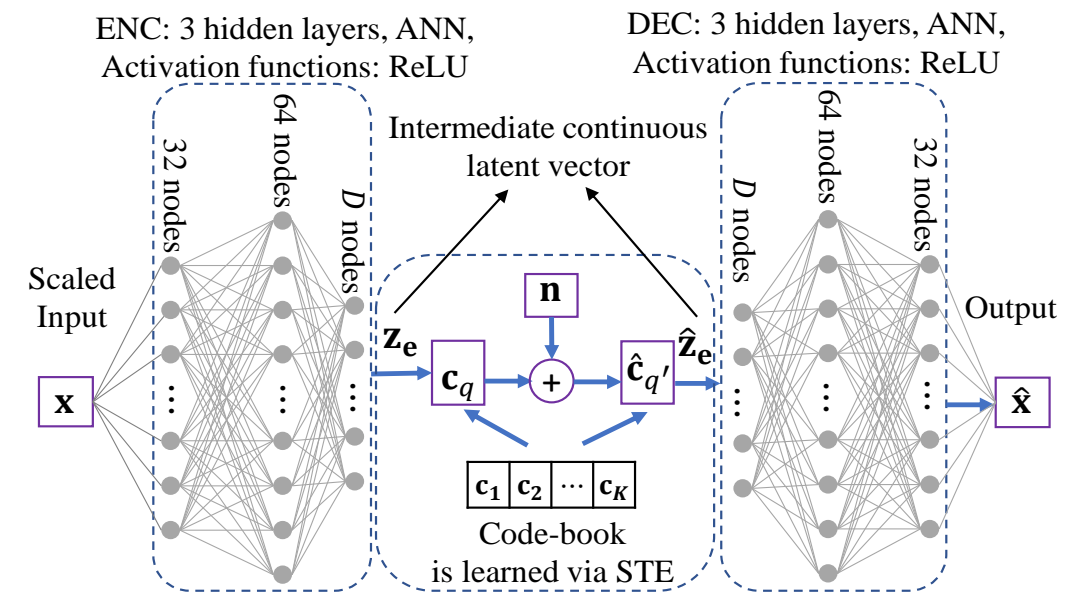

Fig. 2: Training of the noisy system model with the splitted 3-layer ANN ENC and DEC in VQ-VAE.

Since the channel gain, $\alpha$, is random due to fading in wireless communications, the outage probability becomes [10]

$$
p_{\text {out }}(R)=\operatorname{Pr}\left(\log _{2}(1+\gamma(\alpha))<R\right)
$$

which is the probability of unsuccessful decoding. Note that the outage probability in (8) is valid when a capacity-achieving code is used. In practice, since the length of the channel codeword, $M$, may not be long, it would be necessary to consider a finite-length code [7]. In this case, the actual outage probability can be higher than that in (8).

Let $\epsilon=p_{\text {out }}(R)$. Then, from the source coding perspective, the noisy channel can be modeled as a $K$-ary discrete memoryless channel (DMC). In particular, the transition probability of the resulting DMC when $k$ is sent and $k^{\prime}$ is received, is given by

$$
\operatorname{Pr}\left(\hat{\boldsymbol{\kappa}}=k \mid \boldsymbol{\kappa}=k^{\prime}\right)=\left\{\begin{array}{ll}
1-\epsilon, & \text { if } k=k^{\prime} \\
\sum-1 & \text { if } k \neq k^{\prime}
\end{array} .\right.
$$

The VQ-VAE assumes a uniform prior over all the latent codes, so all latents are considered equally likely as in (9). Since there is a one-to-one mapping between $\mathbf{z}$ and $\boldsymbol{\kappa}$, we can see that the received compressed signal after channel decoding, denoted by $\hat{\mathbf{z}}_{e}$, is equal to the compressed signal $\mathbf{z}_{e}$ with a probability of $1-\epsilon$.

Remark: (VQ-VAE Channel Coding Interpretation) Basically, although the convectional VQ-VAE is traditionally used for source coding only, the resilience of the proposed model against noisy channels derives from harmonizing the latent space with the noisy wireless channels. Here, the noisy channel robustness in the deep learning ecosystem is associated with conventional channel coding. Thus, it is considered an integrated source-channel coding.

\section{TRAINING VQ-VAE FOR WiRELESS COMMUNiCATIONS}

Similar to [4], the objective is to minimize the total loss which is composed of three components as follows:

$$
\mathfrak{L}=\overbrace{\mathbb{E}\left[\|\mathbf{x}-\hat{\mathbf{x}}\|^{2}\right]}^{\text {Reconstruction Loss }}+\overbrace{\left\|\operatorname{sg}\left[\hat{\mathbf{z}}_{e}\right]-\mathbf{c}_{k}\right\|_{2}^{2}}^{\text {Quantization Loss }}+\overbrace{\beta\left\|\hat{\mathbf{z}}_{e}-\operatorname{sg}\left[\mathbf{c}_{k}\right]\right\|_{2}^{2}}^{\text {Commitment Loss }},
$$

where function $\mathrm{sg}[$.$] stops the gradient that is defined as identity at forward computation time and has zero partial derivatives$ [4] and coefficient $\beta$ is regularization term for commitment loss. The DEC is trained by optimizing the first loss term, the 


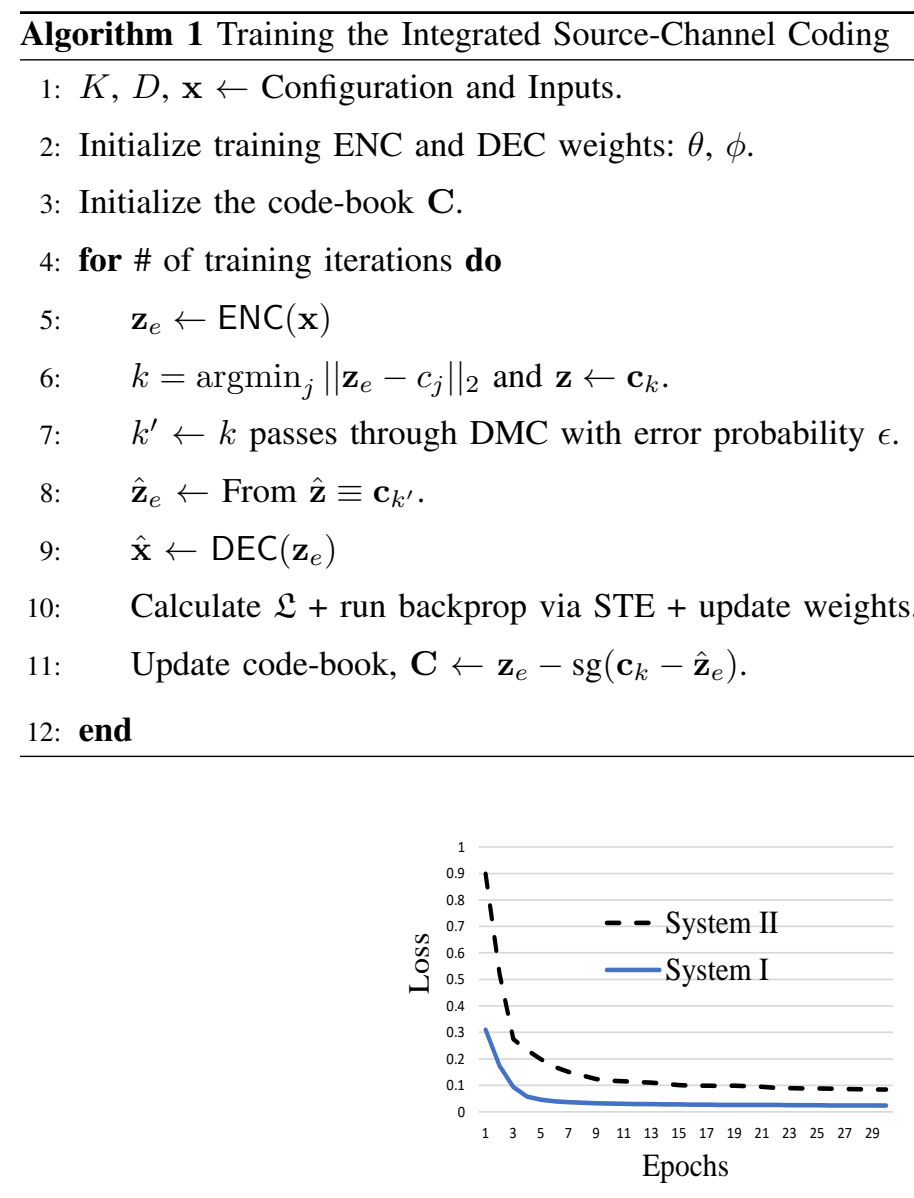

(a) Training Loss

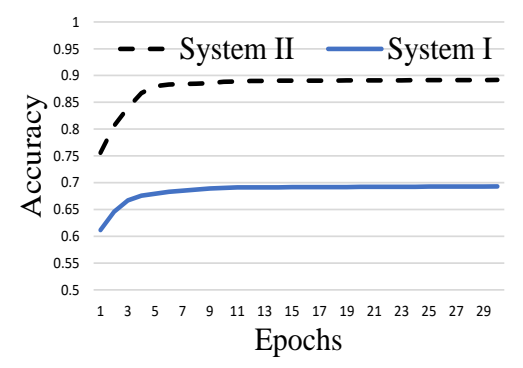

(b) Accuracy in DMC

Fig. 3: Performance of noisy and noise-free trained VQ-VAEs in noisy DMC.

ENC is trained by optimizing the first and final loss terms, and eventually the codewords are learned by optimizing the second and final loss terms [3], [4]. The information theoretic view of the ENC and DEC is briefly given in Appendix. A high-level overview of the training model used in this paper is shown in Fig. 2. Moreover, the training approach is given in Algorithm 1. In the training phase, all we have to do is to feed this algorithm with a dataset $\mathrm{x}$ for the unsupervised learning process. Once it is built with a sufficient number of iterations, the trained ENC and DEC will be used at the Tx and Rx, respectively for an end-to-end integrated source-channel coding.

\section{Experimental Results And Discussions}

We use TensorFlow 2.5 in Python and a MNIST dataset, where every RGB image has size of 28x28 pixels. We apply a straight-through estimator (STE) [3] in between the DEC and the ENC so that the DEC gradients are directly propagated to the ENC. The rest of the parameters used in the experiments are as follows: batch size, epochs, training datasets, test datasets, and $\beta$ are set as $128,30,60000,10000$, and 0.25 , respectively.

Figs. 3 (a) and (b) show the training loss and accuracy of the proposed noisy-trained VQ-VAE, i.e., named System II, compared to the conventional noise-free-trained VQ-VAE, i.e., named System I, where $\epsilon=0.1, K=128$, and $D=16$. Overall, although the training loss is higher in System II (i.e., Fig. 3 (a)), its accuracy in DMC is higher (i.e., Fig. 3 (b)) 


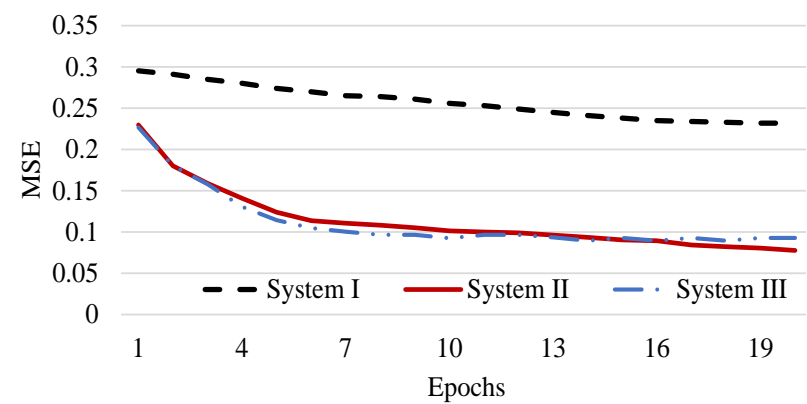

Fig. 4: MSE comparison between the proposed model and conventional repetition coding. $N_{\mathrm{III}}=12, N_{\mathrm{II}}=N_{\mathrm{I}}=4, R=1 / 3$.

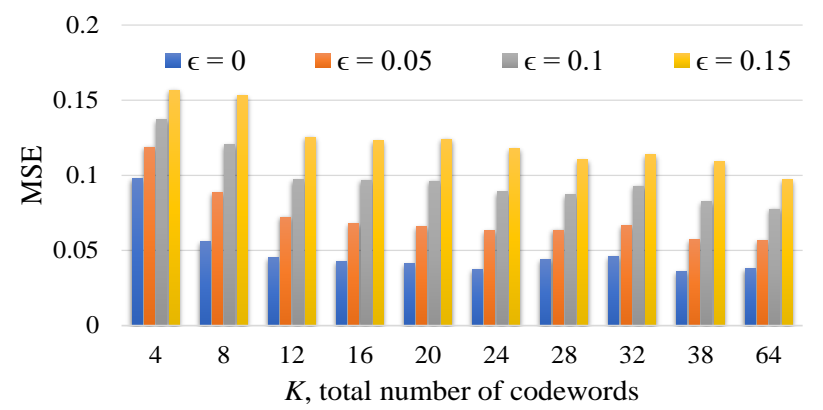

Fig. 5: Rate-distortion plot: impact of $K$ and $\epsilon$ on the MSE of the proposed model for different error probabilities.

compared to System I.

Next, we define System III as the noise-free-trained VQ-VAE but with repetition coding of rate $R=\frac{1}{3}$. For a fair comparison of Systems II and III the number of transmitted bits in System II, $N_{\mathrm{II}}=12$, should be three times more than that in System III, $N_{\text {III }}=4$. As a result, in Fig. 4, we observe that both Systems II and III have relatively similar mean-square-error (MSE) performance and both outperform the System I in a noisy DMC with $\epsilon=0.1$. Indeed, this is the beauty of our key end-to-end integrated source-coding technique, i.e., System II, showing that we can lighter physical/link layer while preserving the system performance.

Fig. 5 illustrates the MSE performance of the proposed model, i.e., System II, where the total number of codewords, $K$, increases and $D=16$. It shows the MSE decreases when $K$ increases. Intuitively speaking, increasing $K$ means increasing the quantization levels which is associated with the latent space resolution. It is also evident that when $\epsilon$ increases the MSE error increases too.

Eventually, Fig. 6 shows the dependency of the system performance on the latent vector dimension, $D$, where $K$ is set 64 . We can observe that the dependency of the MSE on $D$ is minor. This dependency slightly increases when $\epsilon$ increases in low values of $D$. Intuitively, it can also be justified since $D$ refers to the number of nodes in the third and first layer of ENC and DEC, respectively, as shown in Fig. 2. Its impact is supposed to be more when we have less number of hidden layers in the ENC and DEC multilayer perceptrons. 


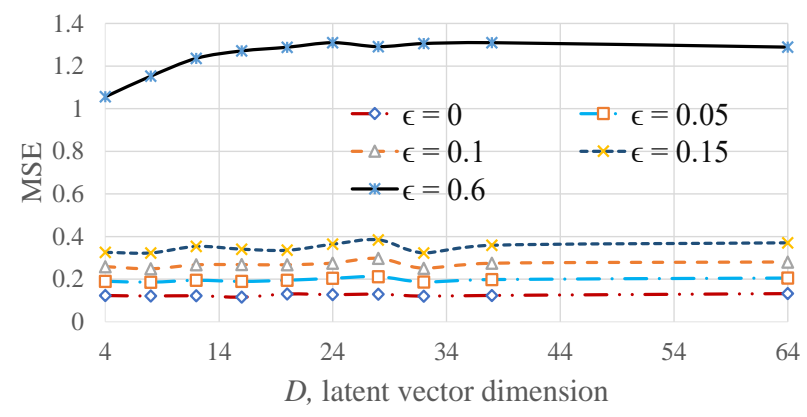

Fig. 6: Impact of latent vector dimension, $D$, on MSE loss.

\section{CONCLUding Remarks}

In this work, we investigated the use of VQ-VAE for joint source-channel coding. We showed the VQ-VAE has characteristics that can be used for digital wireless communications. The proposed data-driven source-channel coding learns the wireless channel variations and becomes robust against noisy channels. We observed that the proposed model makes the physical/link layer lighter while preserving the system performance which is the beauty of our data-driven model. Moreover, it does not require source/channel statistics and the proposed system can be taken into action by enough datasets only. Eventually, we found that the number of latent vectors is associated with the channel coding overhead and its increase results in a better MSE system performance while the dimension of latent vectors has a minor impact on the performance of ENC and DEC multilayer perceptions.

\section{APPENDIX}

\section{Discussion on Prior and Posterior of the LATEnt SPACE}

The VQ-VAE has an artificial NN (ANN) ENC and DEC working together as follows:

$$
\mathbf{x} \rightarrow \mathbf{z} \rightarrow \hat{\mathbf{z}} \rightarrow \hat{\mathbf{x}}
$$

Let $\theta$ and $\phi$ denote the parameters/weights of the ENC and DEC for training, respectively. The ENC is the variational posterior $q_{\theta}(\mathbf{z} \mid \mathbf{x})$. The DEC is a generative model, and it represents the likelihood $p_{\phi}(\mathbf{x} \mid \mathbf{z})$. A joint inference distribution can be defined as

$$
\operatorname{Pr}(\mathbf{x}, \mathbf{z})=\operatorname{Pr}(\mathbf{x}) \operatorname{Pr}(\mathbf{z} \mid \mathbf{x})
$$

The prior of of the latent space, i.e., categorical distribution $\operatorname{Pr}(\mathbf{z})$, can be given as

$$
\begin{gathered}
\int_{\mathbf{x}} \operatorname{Pr}(\mathbf{x}) \operatorname{Pr}(\mathbf{z} \mid \mathbf{x}) d \mathbf{x} \equiv \int_{\mathbf{x}} \operatorname{Pr}(\mathbf{x}, \mathbf{z}) d \mathbf{x} \equiv \operatorname{Pr}(\mathbf{z}) . \\
\text { REFERENCES }
\end{gathered}
$$

[1] C. E. Shannon, "A mathematical theory of communication,” Bell Syst. Tech. J., vol. 27, no. 3, pp. 379-423, 1948.

[2] T. M. Cover and J. A. Thomas, Elements of Information Theory, 2nd ed. NJ: John Wiley, 2006.

[3] A. Hansen Vedal, "Unsupervised Audio Spectrogram Compression using Vector Quantized Autoencoders." Dissertation, 2019.

[4] A. Van Den Oord, O. Vinyals et al., "Neural discrete representation learning," Adv. Neural Inf. Process. Syst., vol. $30,2017$.

[5] J. Choi, Optimal combining and detection: statistical signal processing for communications. Cambridge University Press, 2010. 
[6] - Adaptive and iterative signal processing in communications. Cambridge University Press, 2006.

[7] Y. Polyanskiy, H. V. Poor, and S. Verdu, "Channel coding rate in the finite blocklength regime," IEEE Trans. Inf. Theory, vol. 56, no. 5, pp. 2307-2359, May 2010.

[8] S. B. Wicker, Error control systems for digital communication and storage. Prentice-Hall, Inc., 1995.

[9] A. Guillen i Fabregas and G. Caire, "Coded modulation in the block-fading channel: coding theorems and code construction," IEEE Trans. Inf. Theory, vol. 52, no. 1, pp. 91-114, 2006.

[10] D. Tse and P. Viswanath, Fundamentals of Wireless Communication. Cambridge University Press, 2005. 